\title{
Covid-19 \& Prophylaxis of Ischemic Stroke
}

\author{
Ali MA $^{1}$, Islam $A^{2}$, Chowdhury $\mathrm{RU}^{3}$, Hasan $\mathrm{MS}^{4}$, Rahman $\mathrm{AHMH}^{5}$, Mukherjee $\mathrm{SK}^{6}$, Ashrafee $\mathrm{P}^{7}$
}

Conflict of interest: There is no conflict of interest relevant to this paper to disclose.

Funding Agency : was not funded by any institute or any group.

Contribution of Authors:

Principal Investigator- Col. (Dr.) Md. Amir Ali,

Manuscript preparation -Col. (Dr) Aminul Islam Brig Gen. (Dr) R U Chowdhury.

Data collection- Lt. Col. (Dr) Md. Shafiqul Hasan, Lt. Col. Abu Hasnat Md. Habibur Rahman.

Editorial formatting- Dr. Sudipta Kumar Mukherjee, Dr. Pariza Ashrafee.

Copyright: @2020bang.BJNS published by BSNS. This article is published under the creative commons CC-BY-NC license. This license permits use distribution (https://creativecommons. orgf/licences/by-nc/4-0/)reproduction in any medium, provided the original work is properly cited and is not used for commercial purposes.

Received: $12 / 07 / 2020$

Accepted: $15 / 11 / 2020$

\begin{abstract}
Background: Corona viral disease-19 (COVID-19) is the disease caused by severe acute respiratory syndrome coronavirus 2 (SARS-CoV-2). The whole world is panic about this culprit small virus. It accelerates procoagulation that produce ischemic stroke. Our endeavor is to prevent ischemic stroke. Anti-coagulant and or thrombin inhibitor may be helpful to be prophylactic therapy of ischemic stroke in COVID-19 patients specially with D-dimer positive, severe complications \& co-morbidities.
\end{abstract}

Methods: Total of 76 patients were diagnosed as SARS-CoV-2 and they had been first time reported \& were admitted in Combined Military Hospital (CMH) Mymensingh, within $1^{\text {st }}$ March 2020 to $15^{\text {th }}$ August 2020. Total of 76 cases in either sex \& age variables were randomly selected and divided into two groups one with severe form of disease, procoagulation co-morbidities, coagulopathy-mainly D-dimer positive and another simple COVID-19. Out of 76 patients simple COVID-19 were 41(53.95\%) and complex 35(46.05\%).

Diagnosis was based upon history, clinical examination, and $X$-ray/Computed Tomography (CT) scan of chest and confirmatory by Reverse transcription polymerase chain reaction (RT-PCR).

Results: Total 76 patients COVID-19 were admitted in CMH Mymensingh within $1^{\text {st }}$ March 2020-15 th August 2020 and were evaluated by details history, clinical examination \& relevant investigation.

Here males were 68 (89.47\%); females were 8(10.53\%) \& males were predominant.

COVID-19 incidence among the age group; young adult and adult were higher, 10-19 years 06(7.89\%), 20-29 years 26(34.21\%) and 30-39 years $24(31.58 \%), 40-49$ years $15(19.74 \%)$ and 50 years or more 5(6.58\%) in number. Out of 76 patients, 35(46.05\%) patients were given anticoagulant and thrombin inhibitor therapy. Of that 35 patients $14(40 \%)$ patients were D-dimer positive, $8(22.86 \%)$ patients were cardiovascular disorder (HTN with hypercholesteromia, MI), 5(14.29\%) were DM, severe pulmonary oedema 3(8.57\%) and multiple co-morbidities 5(14.29\%) in number but not given to simple COVID-19 patients.

There were 4 patients who died amongst 76 patients; one with HTN,MI, $2^{\text {nd }}$ one with heart failure, $3^{\text {rd }}$ one with HTN, Old haemorrhagic stroke and last one with CKD and severe pulmonary oedema and mainly they are above 50 years of age and fortunately no one died with ischemic stroke.

Follow- up all patients 02 weeks after SARS-CoV-2 negative and there were no patients suffered from ischemic stroke.

Conclusion: Prevention is always better than cure. Since COVID-19 produce prothrombotic state followed by ischemic stroke, so better to use prophylaxis of ischemic stroke those who are prone; by induce anti-coagulant and or thrombin inhibitor.

Keyword: Corona viral disease-19(COVID-19), Severe acute respiratory syndrome coronavirus 2 (SARS-CoV-2), Reverse transcription polymerase chain reaction (RTPCR), Combined Military Hospital (CMH).

Bang. J Neurosurgery 2021; 10(2): 163-168

1. Col. (Dr.) Md. Amir Ali, Neurosurgeon, CMH Mymensingh.

2. Col. (Dr.) Aminul Islam, Head of Dept. Neurosurgery, CMH Dhaka.

3. Brig. Gen. (Dr) R U Chowdhury, |Advisor Specialist, Neurosurgery, CMH Dhaka.

4. Lt. Col. (Dr) Md. Shafiqul Hasan, Medical Spl, CMH Mymensingh.

5. Lt. Col. Abu Hasnat Md. Habibur Rahman, $\mathrm{CO}, \mathrm{CMH}$, Mymensingh.

6. Dr. Sudipta Kumer Mukherjee, Assistant Prof. Paediatric Neurosurgery, National Institute of Neurosciences and Hospital, Dhaka, Bangladesh.

7. Dr. Pariza Ashrafee, Senior Medical Officer, Ha-meem Group, Dhaka.

Address of Correspondence: Col. Md. Amir Ali, Neurosurgeon, Neurosurgery Center, CMH, Mymensingh, Mobile: +8801742516625 ,

Email: It.col.amirali@gmail.com 


\section{Introduction:}

Simply now the most burning issue in the world is COVID-19. The severe acute respiratory syndrome coronavirus 2 (SARS-CoV-2) has quickly spread worldwide after $1^{\text {st }}$ diagnosed in December 2019, in Wuhan, China ${ }^{1}$.

The outbreak of coronavirus disease 2019 (COVID19) and its pandemicity was declared by the World Health Organization (WHO) in March 11, $2020^{1}$.

COVID-19 due to SARS-CoV-2 virus is continuous changing its genetic material that is mutation till date around thousands time.

COVID-19 primarily affect respiratory system; it may also lead to a hypercoagulable state and thrombotic complications. And recent studies showed that COVID19 might increase the risk of ischemic stroke $e^{2,3,4}$.

The common risk factors for stroke are hypertension (HTN), diabetes mellitus (DM), smoking, alcohol, dyslipidemia, previous stroke and arrhythmia ${ }^{5}$.

Acute cerebrovascular disease (mainly ischaemic stroke) was more with severe COVID-19 than those with non-severe disease ${ }^{6}$.

There is a potential risk for cerebrovascular diseases in patients with COVID-19, with increased risk of morbidity and mortality ${ }^{7,8}$.

The hospitalized patient's mortality rate is higher with COVID-19 and history of stroke compared with COVID19 patients without any history of stroke ${ }^{9}$.

The exact mechanism, phenotype and optimal management of ischaemic stroke associated with COVID-19 are still uncertain. Since there is more chance of ischemic stroke in SARS-CoV-2 virus affected person specially who had coagulopathy, can reduce ischemic stroke by prophylactic anti-coagulant therapy with or without thrombin inhibitor therapy.

As soon as definite coagulopathy or procoagulation evidence confirmed; early therapeutic anticoagulation with LMWH could be beneficial to reduce thromboembolism in patients with COVID-19associated ischaemic stroke. But must it balance against the risk of intracranial haemorrhage, including haemorrhagic transformation of the acute infarct.

\section{Pathogenesis:}

Cerebral stroke mainly two types- ischemic and haemorrhagic, out of them mostly ischaemic stroke; even in COVID-19 related stroke is $90 \%$ ischemic and only $10 \%$ were haemorrhagic stroke ${ }^{9}$.
Ischaemic stroke usually occur due to narrowing of blood vessel lumen by thrombotic mass, or clogging of the same lumens embolus arising from the heart or remote parts of the body.

Cardiac diseases are the frequent source of emboli specially in various cardiac rhythm disorders. Hyperlipidemia is often accompanied with raised blood sugar that increases the degenerative changes in blood vessels. HTN with atherosclerotic changes which produce narrowing of blood vessels followed by reduction of blood supply to brain induce ischemic stroke ${ }^{5}$.

Diabetes mellitus is a metabolic disorder characterized by abnormal glucose metabolism followed by chronic hyperglycemia and also disturbed metabolism of protein and lipid. This phenomenon accelerates atherosclerosis and produce microangiopathy-changes in small vessels like renal, neural and retinal blood vessels, and macroangiopathychanges in large and medium sized blood vessels e.g. the heart, brain and limbs ${ }^{10}$.

Ischemic stroke is mainly due to ischemia of brain by thromboembolic event. COVID-19 associated with stroke yet poorly understood. Hypothesis regarding COVID-19 associated with ischemic stroke-SARSCoV-2 virus infection is associated with vigorous inflammatory response accompanied by coagulopathy, with elevated D-dimer level and presence of antiphospholipid antibodies, which may explain the high prevalence of thrombosis $2,11,12$.

SARS-CoV-2 infection is linked with prothrombotic state, which induces venous and arterial thromboembolic manifestation \& elevated D-dimer level. Severe COVID-19 is associated with proinflammatory cytokines inducing endothelial \& mononuclear cellular activation with expression of tissue factor leading to coagulation activation and thrombin generation. Circulation of free thrombin is uncontrolled by natural anticoagulants, can also activate platelets enhance thrombosis ${ }^{13}$.

COVID-19 with high potency of severe inflammation of respiratory tract followed by extensive bilateral pulmonary oedema, reduced gas exchange, hypoxia induced polycythemia then coagulopathy ${ }^{14}$.

Patients affected with COVID-19 are at risk for medical complications, such as myocardial infarction, heart failure, myocarditis, arrhythmia and venous thrombosis, all of them contribute for ischemic stroke ${ }^{15}$. 
Baseline stroke risk factors, such as DM, HTN and coronary artery disease, were more common in patients with COVID-19; even when adjusting for the number of vascular risk factors, there is higher risk of ischemic stroke with that COVID-19. Other unidentified factors related to COVID-19 may play a role in excess risk of stroke beyond the risk observed in other viral infections is now require further investigation.

\section{Laboratory investigation:}

COVID-19 patients with stroke having higher neutrophil count and lower lymphocyte and platelet count than those without a history of stroke and also interleukin6 , troponin, D-dimer and $\mathrm{N}$-terminal pro-brain natriuretic peptide levels markedly elevated in patients with COVID-19, who had a stroke compared with contemporary COVID-19 patients without stroke symptoms ${ }^{9}$.

Patients with COVID-19 and ischemic stroke at the study, done by Yaghi et al ${ }^{4}$. had not only higher Ddimer level with contemporary COVID-19 patients without stroke, but also elevated troponin and erythrocyte sedimentation rate level, when compared with ischemic stroke patient and patients not infected by SARS-CoV-2 virus.

D-dimer: D-dimer is fibrin degradation product which is small protein in nature and formed in degradation of blood clot by fibrinolysis. It is named as two $D$ fragments of fibrin protein joined by cross-link. Normal value of D-dimer is Â250 $\mathrm{ng} / \mathrm{ml}^{16}$. And D-dimer positive is $\tilde{A} 250 \mathrm{ng} / \mathrm{ml}$ means thrombotic disorder. Elevated D-dimer level is associated with high risk of stroke specially ischemic one and as D-dimer is inexpensive marker and readily accessible; is the great value for prevention of ischemic stroke ${ }^{17}$. SARS-CoV-2 infection is linked with prothrombotic state, which induce thromboembolic manifestation and increase level of D-dimer ${ }^{13}$.

The all patients who were suffering from COVID-19 with ischemic stroke had D-dimer positive and those who had severe form of COVID-19, had elevated ferritin \& $\mathrm{LDH}^{18}$.

The systematic investigation helps for understanding of the mechanism, characteristics and short-term outcome of acute ischemic stroke in patients with SARS-CoV-2 virus infection. It was found that most ischemic stroke occurred in old age, those with traditional stroke risk factors. Initial plasma D-dimer level were 3-fold higher in those who were diagnosed as ischemic stroke than in those who did not ${ }^{15}$.

Lipid profile, blood sugar, HbA1c, ECG with Echocardiogram may require for evaluation of risk of ischemic stroke.

Usually it happens that underestimation occur of the true rate of ischemic stroke, in patients hospitalized with COVID-19 infection because these patients are sometimes too unstable to do brain radio- imaging. Even some patients with undiagnosed COVID-19 and stroke may die before reaching the hospital.

If patients had symptoms and signs of acute ischemic stroke with COVID-19; anti-coagulant, anti-platelet and even interventions, such as thrombolysis and thrombectomy, can be instituted. In the Framingham and Rochester stroke studies, the mortality rate at 30 days after ischemic stroke was $19 \%$, and the 1 year survival rate for patients with ischemic stroke was $77 \%$. Although, the prognosis of acute ischemic stroke varies greatly in individual patients, depending on the stroke severity, age, co-morbidity and poststroke complications ${ }^{19}$.

So at least $23 \%$ casualties occur in ischemic stroke patients at first year; and if conjunction with COVID19 definitely death rate will be higher.

The ischaemic stroke linked to SARS-CoV-2 infection can occur in the context of systemic high prothrombotic state; can support with immediate prophylactic anticoagulation with $\mathrm{LMWH}^{20}$.

In this background to come into conclusion we tried to take preventive measure of those patients with COVID-19 and with prone to ischemic stroke; to see the effects of anticoagulant and thrombin inhibitorin in secondary care hospital like $\mathrm{CMH}$ Mymensingh, Bangladesh.

\section{Materials and methods:}

Our study is a prospective study. All patients with SARS-CoV-2 positive that is COVID-19 who were admitted in $\mathrm{CMH}$, Mymensingh, during the period within $1^{\text {st }}$ March 2020 to $15^{\text {th }}$ August 2020 were enrolled for the study.

Total of 76 cases in either sex \& age variables were randomly selected. These patients were divided into two groups one with coagulopathy-mainly D-dimer positive, a severe form of disease, procoagulation comorbidities, and another simple COVID-19. Out of 76 patients simple COVID-19 were $41(53.95 \%)$ and complex 35(46.05\%). 
Not only Bangladesh whole world is now in severely crisis of corona disease. All patients were analyzed and evaluated in our hospital. Clinical, biochemical, RT-PCR, radiological data of patient; recorded from hospital record, picture archive and communication system. Patients with SARS-CoV-2 positive with or without any co-morbidity were included. All of them were RT-PCR sorted and included in this study. Patients of any age group with irrespective of gender, co-morbidities such as DM, HTN, hypercholesteromia, $\mathrm{Ml}$; on anticoagulant, coagulopathy etc. were included in this study. Evaluation of condition of the patients by history, clinical examination and investigation after treatment until corona virus is negative (by RT-PCR) and was compared with / without treatment of anticoagulant and thrombin inhibitor of $D$-dimer positive, multiple comorbidities or severe form of disease like severe pulmonary oedema, prolong hypoxia and simple COVID-19. They also followed-up, 02 weeks after RT-PCR negative.

\section{Results:}

Total 76 patients COVID-19 who were admitted in $\mathrm{CMH}$ Mymensingh within 1st March 2020-15 th August 2020. All of patients were evaluated by details history, clinical examination \& relevant investigation.

Regarding gender, males were 68 (89.47\%); females were $8(10.53 \%)$ \& males were predominant (Figure-1).

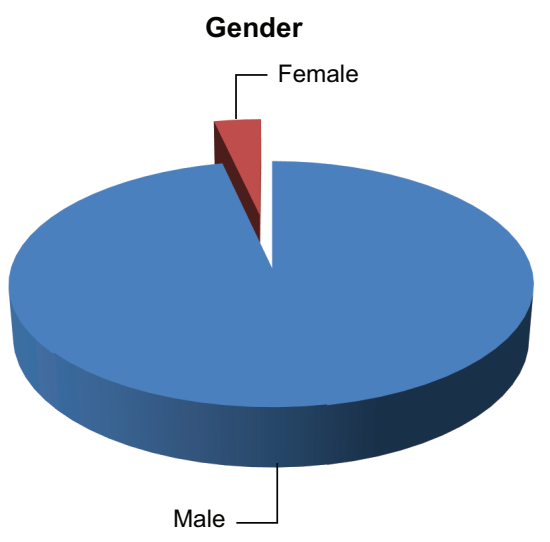

Fig.-1:

COVID-19 incidence among the age group; $10-19$ years $06(7.89 \%), 20-29$ years $26(34.21 \%$ ) and $30-39$ years $24(31.58 \%), 40-49$ years $15(19.74 \%)$ and 50 years or more $5(6.58 \%)$ in number. So young and young adult are more in number (Figure-2)

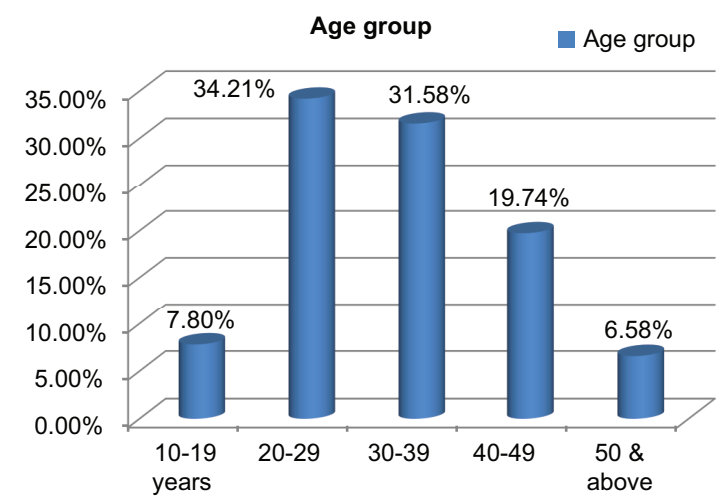

Fig: 2

Anticoagulant and or thrombin inhibitor therapy was induced in D-dimer positive, severe hypoxia usually due to severe pulmonary oedema and severe co-morbid patients. Out of 76 patients $35(46.05 \%$ ) patients were given anticoagulant and or thrombin inhibitor therapy. Of that 35 patients, $14(40 \%)$ patients were D-dimer positive, $8(22.86 \%)$ patients were cardiovascular disorder (HTN with hypercholesteromia, MI), 5(14.29\%) were DM, severe pulmonary oedema $3(8.57 \%)$ and multiple co-morbidities 5(14.29\%) in number. But not induce anticoagulant and or thrombin inhibitor therapy to simple COVID-19 patients. (Figure-3)

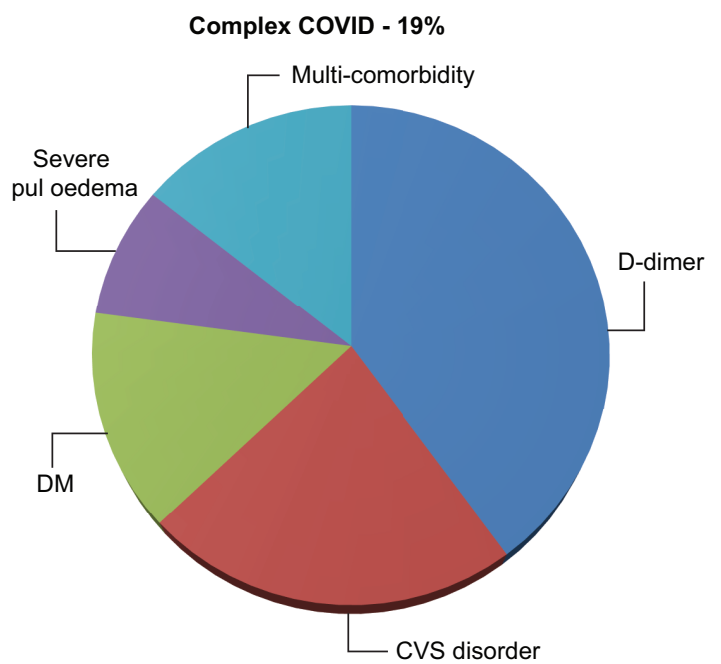

Fig.-3:

Out of 76 patients 4 patients were died; one with HTN, MI, $2^{\text {nd }}$ one with heart failure, $3^{\text {rd }}$ one with HTN, Old haemorrhagic stroke and last one with CKD and severe pulmonary oedema and mainly they are above 50 years of age and fortunately no one died with ischemic stroke. 
We have followed up all patients 02 weeks after RTPCR negative and there were no patients suffered from ischemic stroke.

\section{Discussion:}

Regarding anticoagulant and thrombin inhibitor therapy in COVID-19 patients we have seen those who are D-dimer positive, severe hypoxia usually due to pulmonary oedema and having severe co-morbidities like DM, hypercholesteromia usually associated with HTN that are prone to coagulopathy- thrombosis ; anticoagulant with thrombin inhibitor are most useful prophylactic drugs. Usually we used clexane S/C, 60 mg BD for 07 days and Rivaroxaban 10 mg BD for several weeks. We followed-up all the patients after 02 weeks of RT-PCR negative, no one developed ischemic stroke both given anti-coagulant \& thrombin inhibitor and not given those in simple COVID-19 disease.

Some study shown COVID-19 is primarily affect respiratory system; it also lead to a hypercoagulable state and thrombotic complications ${ }^{2,3,4}$.

New York stroke center raising concerns for a potential risk for severe stroke syndromes in young patients affected by COVID-1921.

According to Alexander $\mathrm{E}$ et $\mathrm{al}^{15}$., of COVID-19 affected population; the median age was 64 years ( $51-76$ years), $57 \%$ were men, and $43 \%$ were female.

In another study by Shadi Yaghi et al ${ }^{4}$., COVID-19 positive patients were more likely to be younger men.

According to $\mathrm{WHO}$, in Palestine most affected population is women $54.78 \%$ and man $45.22 \%$ and mostly affected age group is $18-29$ years $35.65 \%{ }^{22}$.

In our study males were 68 (89.47\%); females were $8(10.53 \%)$ in number \& males were predominant which is similar to the study of Shadi Yaghi et al., as our soldiers are mostly male personnel.

In this study of CMH Mymensingh; COVID-19 incidence among age group, $10-19$ years $06(7.89 \%)$, $20-29$ years $26(34.21 \%)$ and $30-39$ years $24(31.58 \%)$, $40-49$ years $15(19.74 \%)$ and 50 years or more $5(6.58 \%)$ in number that is young and young adult are affected more in number; which is similar to the study of WHO in Palestine as our serving soldiers are mostly young and young adult.

D-dimer is usually elevated in patients with COVID19 and it also correlates severity of disease expressed by $\mathrm{Yao} Y$ et $\mathrm{al}^{23}$.
According to Alexander $\mathrm{E}$ et $\mathrm{al}^{15}$., initial plasma Ddimer levels were around 3-fold higher in those who were diagnosed ischemic stroke than in those who did not so.

In our study, out of total 76 patients of COVID-19, $14(18.42 \%)$ were D-dimer positive and those 35 patients who were complex variety and given anticoagulant and thrombin inhibitor therapy, D-dimer raised $14(40 \%)$ which is similar to Yao, $Y$ et al., study.

Among the COVID-19, 0.9\% had an ischemic stroke stated by Yaghi S et al ${ }^{4}$., in May 2020.

Other recent studies suggesting that stroke is increased with a 2.5- fold in severe COVID-19, with increased mortality rate ${ }^{24}$.

The patients with SARS-CoV-2 infection who experienced an ischemic stroke were considerably more mortile than patients with COVID-19 infection who had not an ischemic stroke ${ }^{15}$.

Therapeutic anticoagulation in patients with high Ddimer level may lead to a lower rate of thrombotic complications including ischemic stroke in hospitalized COVID-19 positive patients ${ }^{4}$.

All patients had large vessel occlusion with markedly elevated D-dimer levels (e"10001/4g/L). Out of them three patients had multiple infarcts, two had concurrent venous thrombosis, and in two ischaemic stroke occurred despite therapeutic anticoagulant ${ }^{25}$.

According to to J. Djelilovic-Vranic et al ${ }^{5}$., the common risk factors for stroke are hypertension, diabetes mellitus, smoke, alcohol, dyslipidemia, previous stroke and arrhythmia.

There is more chance of ischemic stroke as well as death in patients with $\mathrm{D}$-dimer positive, procoagulation factors like diabetes mellitus, dyslipidemia with hypertension, MI, arrhythmia, hypoxia(pulmonary oedema) are accelerated by COVID-19. And we treated those patients with anti-coagulant and thrombin inhibitor therapy without delay and no one suffered from ischemic stroke in our study.

In 2020, Zhang $Y$ et al ${ }^{2}$., showed that SARS-CoV-2 usually stimulate to produce antiphospholipid antibodies (aPL) as a mechanism of ischaemic stroke but post infection aPL are transient and rarely associated with thrombosis.

We have followed-up the patients 02 weeks after SARS-CoV-2 negative and there were no patients suffered from ischemic stroke which is as the study of Zhang Y et al., study. 
There are some limitations of this study - it was done in single center, $\mathrm{CMH}$ Mymensingh and less in number of patients and short duration of study. But as whole country affected by COVID-19 and our patients are entitle both serving (lives different parts of the country), retired soilders \& parents of our military members and reported to our $\mathrm{CMH}$ Mymensingh, it can reflects some overall scenario of at least Bangladesh.

\section{Conclusion:}

We all know that prevention is always is better than cure. Since COVID-19 produce prothrombotic state followed by ischemic stroke, so better to prevent ischemic stroke those who are prone; by induce anticoagulant and or thrombin inhibitor by keeping mind chance of ischemic stroke. And thereby reduce burden of long term disability and mortality.

\section{References:}

1. Zhu N, Zhang D, Wang W, Li X, Yang B, Song J, Zhao X, Huang B, Shi W, Lu R, et al. A novel coronavirus from patients with pneumonia in China, 2019. N Engl J Med. 2020; 382:727-733.

2. Zhang $\mathrm{Y}$, Xiao M, Zhang S, et al. Coagulopathy and antiphospholipid antibodies in patients with COVID19. ÿp N Engl J Med. 2020; 382(17):e38.

3. Klok FA, Kruip MJHA, van der Meer NJM, et al. Incidence of thrombotic complications in critically ill ICU patients with COVID-19. ÿp Thromb Res. 2020; 191(191):145-147.

4. Yaghi S, Ishida K, Torres J, et al. SARS2-CoV-2 and stroke in a New York healthcare system. ÿp Stroke 2020; A120030335.

5. Djelilovic-Vranic J, Alajbegovic A et al. Predilection Role Diabetes Mellitus and Dyslipidemia in the Onset of Ischemic Stroke. Med Arh. 2013 Apr; 67(2): 120-123.

6. Mao L, Jin H, Wang M, et al. Neurologic manifestations of hospitalized patients with coronavirus disease 2019 in Wuhan, China. JAMA Neurol. 2020.

7. Helms J, Tacquard C, Severac F, Leonard-Lorant I, Ohana $M$, Delabranche X, Merdji H, Clere-Jehl R, Schenck M, Gandet FF, et al. High risk of thrombosis in patients with severe SARS-CoV-2 infection: a multicenter prospective cohort study. Intensive Care Med. 2020; 1-10.

8. Klok FA, Kruip MJHA, van der Meer NJM, Arbous MS, Gommers D, Kant KM, Kaptein FHJ, van Paassen J, Stals MAM, Huisman MV, Endeman $\mathrm{H}$. Confirmation of the high cumulative incidence of thrombotic complications in critically ill ICU patients with COVID19: an updated analysis. Thromb Res.2020; pii: S00493848(20)30157-2.

9. Qin C, Zhou L, Hu Z, Yang S, Zhang S, Chen M, Yu H, Tian DS, Wang W. Clinical characteristics and outcomes of COVID-19 patients with a history of stroke in Wuhan, China. Stroke 2020; 51:2219-2223.

10. Gamulin S, Maruši M, Kova Z. i saradnici, Patofiziologija, šesto, obnovljeno i izmijenjeno izdanje, Knjiga prva, Medicinska naklada; Zagreb 2005: 170-212.
11. Spiezia L, Boscolo A, Poletto F, et al. COVID-19-related severe hypercoagulability in patients admitted to intensive care unit for acute respiratory failure. Thromb Haemost. 2020; 120(6):998-1000.

12. Panigada M, Bottino N, Tagliabue $P$, et al. Hypercoagulability of COVID-19 patients in intensive care unit: a report of thromboelastography findings and other parameters of hemostasis. J Thromb Haemost. 2020.

13. Tang N, Li D, Wang X, et al. Abnormal coagulation parameters are associated with poor prognosis in patients with novel coronavirus pneumonia. J Thromb Haemost. 2020; 18:844-7.

14. Zangari $\mathrm{M}$ et al.could hypoxia increase the prevalence of thrombotic complications in polycythemia vera? NIH-PA Author Manuscript 2013 April; 24(3):311-31.

15. Alexander E, Merkler.Neal S, Parikh et al. Risk of Ischemic Stroke in Patients with Coronavirus Disease 2019 (COVID19) vs Patients with Influenza. JAMA Neurol. 2020 July 2

16. Pagana KD, Pagana TJ, Pagana TN, Mosbys Diagnostic \& Laboratory Test Reference. $14^{\text {th }}$ ed.St.Louis, Mo: Elsevier; 2019.

17. Zakai NA, McClure LA, Judd SE,Kissela b,Howard G,Ssfford M,Cushman M. D-dimer and the Risk of Stroke and Coronary Heart Disease. The Reasons for geographic and Racial Differences in Stroke (REGARDS) study. Thromb haemost. 2017; 117:618-24.

18. Rahma B, Matthew E Adams et al. Characteristics of ischaemic stroke associated with COVID-19. J Neurol Neurosurg Psychiatry 2020; 91:889-891.

19. Adams HP. Davis PH. Leira EC.Chang KC.Bendixen BH. Clarke WR. Woolson RF. Hansen. Baseline NIH Stroke Scale score strongly predicts outcome after stroke: A report of the Trial of Org 10172 in Acute Stroke Treatment (TOAST). Neurology. 1999; 53(1):126-31.

20. Thachil J, Tang N, Gando S, et al. ISTH interim guidance on recognition and management of coagulopathy in COVID19. Journal of Thrombosis and Haemostasis 2020; 18:1023-26

21. Oxley TJ, Mocco J, Majidi S, Kellner CP, Shoirah H, Singh IP, De Leacy RA, Shigematsu T, Ladner TR, Yaeger KA, et al. Large-vessel stroke as a presenting feature of COVID19 in the young. N Engl J Med.2020; 382:e60.

22. https://who18.createsend.com/campaigns/reports/view Campaign. aspx $\mathrm{d} d=\mathrm{j} \& \mathrm{c}=$ 99FA4938D049 E3A8\&ID= OF7F4F328 EA22F6C2540 EF23F30FEDED \& temp $=$ False $\& \mathrm{t} x=0 \&$ source $=$ Report.

22. https://who18.createsend.com/campaigns/reports/view Campaign. aspx?d=j\&c= 99FA4938D049 E3A8\&ID= OF7F4F328 EA22F6C2540 EF23F30FEDED \& temp= False \& $\mathrm{t} x=0$ \&source $=$ Report

23. Yao Y, Cao J, Wang Q et al. D-dimer as a biomarker for disease severity and mortality in COVID-19 patients: a case control study. J intensive care 2020; 49:8.

24. Aggarwal G, Lippi G, Michael Henry B. Cerebrovascular disease is associated with an increased disease severity in patients with coronavirus disease 2019 (COVID-19): a pooled analysis of published literature. Int J Stroke 2020.

25. Beyrouti R, Adams ME, Benjamin Characteristics of ischaemic stroke associated with COVID-19. J Neurol Neurosurg Psychiatry 2020 April 27:1-3. 